\title{
Changes in photosynthetic characteristics and photosystem stoichiometries in the lower leaves in rice seedlings
}

\author{
Jun-ya Yamazaki $^{\text {a,*, }}$ Yasumaro Kamimura ${ }^{a}$, Mitsumasa Okada ${ }^{\text {, }}$ \\ Yasutomo Sugimura ${ }^{\text {a }}$ \\ a Department of Biology, Faculty of Science, Toho University, 2-2-1 Miyama, Funabashi, Chiba 274-8510, Japan \\ ${ }^{\mathrm{b}}$ Department of Biomolecular Science, Faculty of Science, Toho University, 2-2-1 Miyama, Funabashi, Chiba 274-8510, Japan
}

Received 5 April 1999; received in revised form 7 July 1999; accepted 7 July 1999

\begin{abstract}
Changes in the Chl $a / b$ ratio, electron transport, electron carriers, and photosystem stoichiometries were examined in rice leaves in the present study. The $\mathrm{Chl} a / b$ ratio is known to decrease gradually from the top to the bottom leaves, indicating a increase in the abundance of LHC II relative to the reaction center complexes of the two photosystems. We used juvenile rice canopy and obtained the following results: (1) the photosynthetic activity and Chl content per leaf area decreased from the top to the bottom leaves, the Chl $a / b$ ratio also declined from 3.7 to 3.0 ; (2) when determined on the basis of Chl content, C-550 and Cyt $f$ content decreased, but there was no loss of P-700, Consequently, the PS II/PS I ratio significantly decreased; (3) on the basis of mmol Chl, the levels of Cyt $f$ dramatically decreased and, therefore, no loss was observed for whole chain electron transport per Cyt $f$; and (4) the percentage abundance of PS II $\alpha$ increased, but the rate constants of $Q_{\mathrm{A}}$ photoreduction and P-700 photooxidation gradually decreased. From these results, we hypothesize that there is a compensatory relationship between the decline in the $\mathrm{Chl}$ $a / b$ ratio and that in the PS II/PS I ratio in the lower leaves in rice seedlings. (C) 1999 Elsevier Science Ireland Ltd. All rights reserved.
\end{abstract}

Keywords: Antenna size of PS I and PS II; Chl $a / b$ ratio; Light intensity; PS II heterogeneity; PS II/PS I ratio; Rice

\section{Introduction}

Light is an important factor for plant growth. In vertically vascular plants, because leaves that develop at the top of a canopy receive full sunlight, lower leaves receive very weak light. Accordingly, the light intensity decreases exponentially from the uppermost to the bottom layer made by mutual shading of leaves [1]. Hikosaka [2] and Okada and Katoh [3] made several important

\footnotetext{
Abbreviations: C-550, electrochromic shift of pheophytin in the reaction center complex of photosystem II; Chl, chlorophyll; Cyt, cytochrome; DCIP, 2,6-dichlorophenolindophenol; DCMU, 3-(3,4dichlorophenyl)-1,1-dimethylurea; LHC I and II, the light-harvesting chlorophyll $a / b$-protein complex in PS I and II, respectively; P-700, the reaction center complex in photosystem I; PS, photosystem; $Q_{\mathrm{A}}$, primary quinone acceptor of PS II.

* Corresponding author. Tel./fax: + 81-47-472-5362.

E-mail address: junya@bio.sci.toho-u.ac.jp (J.-y. Yamazaki)
}

statements on the aggravation of the light environment, especially the attenuation of the light intensity. They claimed that photosynthesis in lower leaves is limited by light intensity, and an excessive amount of the proteins related to $\mathrm{CO}_{2}$ fixation are degraded rapidly. In contrast, the proteins involved in the harvesting and utilization of light are effectively protected in weak light [3], as in the example of the decline in the ratio of Chl $a$ to $b$ during leaf senescence [4]. A decline in the Chl $a / b$ ratio indicates a more rapid degradation of the reaction center complexes of the photosystems than of LHC II [4-6]. Thus, lower leaves retain the capacity to drive photosynthesis maximally under the light-limiting conditions of shade. This indicates that a plant has the plasticity to adjust its photosynthetic apparatus in response to the appearance of mutual shading leaves that are inevitably associated with plant growth. 
There are many reports that the spectral quality and/or the intensity of light affect the changes in the Chl $a / b$ and the PS II/PS I ratios [5-11]. It should be noted that most of these findings were obtained from experiments in which plants were grown in artificially controlled light conditions. Little is known about these changes when plants were grown under natural conditions. Recently, the PS II/PS I ratio is less affected by natural shade compared with artificial conditions where the light intensity and spectral quality conditions can be extreme [10].

In several reports about photosystem stoichiometries, Melis and his associates [11,12] reported on the effects of light quality during plant and algal cell growth, and they revealed a compensatory system adjusting the two photosystem stoichiometries depending on light quality by using spectrophotometrical analysis. In contrast, a few reports examined the effects of light intensity using spectrophotometric measurement [7].

In terms of both function and antenna size, the PS II reaction center is heterogeneous $[12,13]$. One center has a large antenna and is functional in electron transport, and one has a small antenna and is unable to transfer electrons from $Q_{\mathrm{A}}^{-}$to $Q_{\mathrm{B}}$. The former is defined as PS II $\alpha$, and the latter as PS II $\beta$. As a result of the large difference in antenna size, photoreduction of $Q_{\mathrm{A}}$ in PS II $\alpha$ proceeds more rapidly than it does in PS II $\beta$ under irradiation with a limited-intensity green light in the DCMU-poisoned thylakoids.

In the present study, the $\mathrm{Chl} a / b$ ratio, levels of the reaction center complex of PS I and PS II and of Cyt $b_{6} / f$ complex, and electron transport activity were determined at the different leaf positions of rice seedlings. In addition, the relative antenna sizes of PS I and PS II were determined by measuring the kinetics of photooxidation of P-700 and photoreduction of $Q_{\mathrm{A}}$. Using the results obtained, we discuss herein the physiological significance of a decline in the $\mathrm{Chl} a / b$ ratio in the lower leaves.

\section{Materials and methods}

\subsection{Plant growth conditions}

Rice plants (Oryza sativa L. cv. Nipponbare) were grown in a greenhouse for 4-5 weeks as described in [4]. Germinated seeds were planted at 3-cm intervals in an artificial granular soil, Bonsol No. 1 (Sumitomo Kagaku, Osaka, Japan) that contained sufficient levels of nutrients for the growth of rice seedlings. The plants were watered daily. Leaves were numbered in the order of their development. Monocotyledonous rice leaves successively developed with high synchronization, and a newly developed leaf blade was always longer than a lower leaf blade that had developed earlier. The 3rd-6th leaves were harvested immediately after full expansion of the 6th leaves had occurred. By this time, the 1st and 2nd leaves had withered and the tips of the 3rd leaves had turned yellow.

\subsection{Light intensity and spectral quality measurements}

Light intensity was measured at each leaf position with a Li-Cor model LI-189 light meter (Lincoln Co., Lincoln, NE, USA). Spectral photon distribution measurements were made for each leaf position using a Li-Cor model LI-1800 spectroradiometer. The sensor probe was placed on the adaxial surface of the center leaf blade to evaluate the transmission of light through the upper leaves. Each measurement was the average of three spectral scans taken from 300 to $800 \mathrm{~nm}$ at $2-\mathrm{nm}$ intervals. Light intensity and spectral photon distributions were measured between 1300 and 1500 $\mathrm{h}$ on cloudless, clear days.

\subsection{Measurement of photosynthesis}

Photosynthesis of leaves was measured with a Hansatech leaf-disc oxygen electrode at $30^{\circ} \mathrm{C}$. The gas phase consisted of air containing $4 \% \mathrm{CO}_{2}$. A saturating actinic light $\left(2000 \mu \mathrm{mol}\right.$ photons $\mathrm{m}^{-2}$ $\mathrm{s}^{-1}$ ) from a $100-\mathrm{W}$ halogen lamp passed through a Hoya HA heat-absorbing filter. Leaf area was estimated by inputting leaf shapes into a microcomputer (NEC PC-9801 RX) with an image scanner (EPSON GT-6500).

\subsection{Isolation of thylakoid membranes and their photosynthetic activities}

The thylakoid membranes of rice leaves were isolated as described in [4]. In brief, leaves were homogenized in a cold buffer that contained $0.4 \mathrm{M}$ sucrose, $10 \mathrm{mM} \mathrm{NaCl}, 5 \mathrm{mM} \mathrm{MgCl}_{2}$, and $50 \mathrm{mM}$ HEPES-NaOH (pH 7.5) for $30 \mathrm{~s}$ with a Waring 
blender. The homogenate was filtered through two layers of Miracloth (Calbiochem, USA), and the filtrate was centrifuged at $250 \times g$ for $5 \mathrm{~min}$. The supernatant was centrifuged at $5500 \times g$ for 15 min, and the precipitate was suspended in the medium just described. The rates of evolution or consumption of oxygen at light-saturation were measured with a Clark-type oxygen electrode at $25^{\circ} \mathrm{C}$ in a basal medium that contained $0.4 \mathrm{M}$ sucrose, $10 \mathrm{mM} \mathrm{NaCl}, 5 \mathrm{mM} \mathrm{MgCl}_{2}$, and $50 \mathrm{mM}$ HEPES-NaOH ( $\mathrm{pH}$ 7.5). PS II activity was measured by adding $1 \mathrm{mM}$ phenyl- $p$-benzoquinone to a basal reaction medium. PS I activity was determined in the presence of $10 \mu \mathrm{M}$ DCMU, $500 \mu \mathrm{M}$ DCIP, $1 \mathrm{mM}$ methyl viologen, and $2 \mathrm{mM}$ sodium ascorbate. For whole chain electron transport, 1 $\mathrm{mM}$ methyl viologen and $1 \mathrm{mM} \mathrm{NaN}$ were added to the basal medium. All mixtures contained 20 $\mathrm{mM} \mathrm{HCl-methylamine} \mathrm{as} \mathrm{an} \mathrm{uncoupler,} \mathrm{and} \mathrm{thy-}$ lakoid membranes were adjusted to $11 \mu \mathrm{M} \mathrm{Chl}$. Chlorophyll was determined by the method of [14].

\subsection{Determination of PS I and PS II reaction center contents}

P-700 and C-550 were measured with a Hitachi 556 dual-wavelength spectrophotometer. P-700 was determined by measuring light-induced absorbance changes at $700 \mathrm{~nm}$ with a reference wavelength at $730 \mathrm{~nm}$. The reaction medium contained $50 \mathrm{mM}$ HEPES-NaOH (pH 7.5), $10 \mathrm{mM}$ $\mathrm{NaCl}, 5 \mu \mathrm{M}$ DCIP, $1 \mathrm{mM}$ sodium ascorbate, 10 $\mu \mathrm{M}$ DCMU, $1 \mathrm{mM}$ methyl viologen, $0.05 \%(\mathrm{w} / \mathrm{v})$ Triton X-100, and thylakoid membranes equivalent to $11 \mu \mathrm{M}$ Chl. Actinic light passed through a blue band-pass filter (Corning, CS 4-96), and the photomultiplier was protected with a red cut-off filter (Toshiba, R-66). The differential absorption coefficient of P-700 was taken to be $64 \mathrm{mM}^{-1}$ $\mathrm{cm}^{-1}$ [15].

Light-induced absorbance changes of C-550, an electrochromic band shift of pheophytin that is associated with $Q_{\mathrm{A}}$ photoreduction [16], was determined at $550 \mathrm{~nm}$ with a reference wavelength at $540 \mathrm{~nm}$ as described previously [17]. Red actinic light was obtained by passing light through a 650-nm interference filter, with the photomultiplier guarded by a CS 4-96 filter. The differential absorption coefficient used was $5.2 \mathrm{mM}^{-1} \mathrm{~cm}^{-1}$ [17]. The reaction medium contained $50 \mathrm{mM}$
HEPES-NaOH (pH 7.5), $10 \mathrm{mM} \mathrm{NaCl}, 15 \mu \mathrm{M}$ DCMU, $20 \mu \mathrm{M}$ gramicidin D, $2 \mathrm{mM}$ ferricyanide, $0.1 \%(\mathrm{w} / \mathrm{v})$ Triton X-100, and thylakoid membranes $(82 \mu \mathrm{M} \mathrm{Chl})$. The detergent treatment effectively removed the light-scattering, and we made sure that this concentration of the detergent did not influence the measurement of C-550. We also made sure that the actinic light used for P-700 and C-550 determination was sufficiently saturating for the measurement. Cyt $f$ contents were determined with a Hitachi 556 spectrophotometer in a double-beam mode according to the method described in [18]. The reaction mixture contained $50 \mathrm{mM}$ HEPES-NaOH ( $\mathrm{pH}$ 7.5), $10 \mathrm{mM} \mathrm{NaCl}$, $1 \%(\mathrm{w} / \mathrm{v})$ Triton $\mathrm{X}-100$, and thylakoid membranes (45 $\mu \mathrm{M}$ Chl). The differential absorption coefficient used was $17.7 \mathrm{mM}^{-1} \mathrm{~cm}^{-1}$ [18].

\subsection{Relative antenna size measurement}

The area over the fluorescence induction curve was measured with a laboratory-constructed apparatus. Green light obtained by passing light from a halogen lamp (Hayashi Tokei Kogyo, LA150SE) through a CS 4-96 filter and a Toshiba O-54 cut-off filter was used for excitation. This region was used in order to excite equally both $\mathrm{Chl}$ $a$ and $\mathrm{Chl} b$ molecules. Actinic light intensity was varied with Toshiba neutral-density filters. Fluorescence was monitored at $690 \mathrm{~nm}$ by inserting a Toshiba R-64 red cut-off filter and a high-intensity Bausch \& Lomb grating monochrometer between the sample cuvette and the photomultiplier (Hamamatsu Photonics, R928). Signals were stored in a transient recorder (Riken Denshi, TCDC-12-8000(E)) and analyzed with a computer. The two types of PS II reaction centers, the PS II $\alpha$ and PS II $\beta$ centers, were estimated by analyzing fluorescence induction curves in the presence of DCMU as described in [19,20]. The reaction medium contained $50 \mathrm{mM}$ HEPES-NaOH $(\mathrm{pH}$ 7.5), $10 \mathrm{mM} \mathrm{NaCl}, 5 \mathrm{mM} \mathrm{MgCl} 2,20 \mu \mathrm{M}$ DCMU, and $0.4 \mathrm{M}$ sucrose and thylakoid membranes (11 $\mu \mathrm{M}$ Chl).

The P-700 photooxidation kinetic measurements were performed according to [21] with the slight modifications described in [22]. In brief, the thylakoid membranes $(110 \mu \mathrm{M} \mathrm{Chl})$ were incubated in $50 \mathrm{mM}$ HEPES-NaOH (pH 7.5) and $150 \mathrm{mM}$ $\mathrm{KCN}$ for $2 \mathrm{~h}$ on ice, to block electron transport at plastocyanin. After incubation, the samples were 
diluted tenfold in a medium contained $50 \mathrm{mM}$ HEPES-NaOH ( $\mathrm{pH}$ 7.5), $10 \mathrm{mM} \mathrm{NaCl}, 5 \mathrm{mM}$ $\mathrm{MgCl}_{2}, 0.4 \mathrm{M}$ sucrose, $20 \mu \mathrm{M}$ DCMU, and 200 $\mu \mathrm{M}$ methyl viologen. The color and intensity of the actinic light were the same as that in a measurement of the fluorescence induction kinetics. Signals were stored in a transient recorder and analyzed with a computer.

\section{Results and discussion}

In this study, we investigated the changes in photosynthetic characteristics with depth of canopy in rice seedlings. Photosynthesis, Chl levels, and the Chl $a / b$ ratio of the $3 \mathrm{rd}-6$ th leaves are shown in Table 1. The rates of light-saturated photosynthesis $\left(P_{\max }\right)$ decreased with the depth of the canopy except for the 5th leaves, which showed a $P_{\max }$ similar to that of the 6th leaves. Chl levels were highest in the 5th leaves, and then decreased from the top to the bottom leaves. This may indicate that the 6th leaves contain some developing photosynthetic apparatus. As shown in Table 1, the Chl $a / b$ ratio decreased gradually from 3.7 in the 6 th leaves to 3.0 in the 3rd leaves. Although leaf senescence is primarily under genetic control, it is well established that the light environment of leaves is a major factor in the modulation of the Chl $a / b$ and PS II/PS I ratios [2,3]. The changes in the $\mathrm{Chl} a / b$ and PS II/PS I ratios that occur during senescence of erect herbaceous canopy leaves must be caused mainly by shading of these leaves by upper leaves [2,3].

Levels of the PS II and PS I reaction center complexes were also affected by the light intensity $[8,10,23,24]$. These levels were quantified by measuring light-induced absorbance changes of

Table 1

Photosynthetic capacities, $\mathrm{Chl} a / b$ ratio and levels of $\mathrm{Chl}$ in the 3 rd-6th leaves of the juvenile rice seedlings ${ }^{\text {a }}$

\begin{tabular}{llll}
\hline Leaf position & $\begin{array}{l}P_{\max }(\mu \mathrm{mol} \\
\left.\mathrm{O}_{2} \mathrm{~m}^{-2} \mathrm{~s}^{-1}\right)\end{array}$ & $\begin{array}{l}\mathrm{Chl}(\mu \mathrm{mol} \\
\left.\mathrm{m}^{-2}\right)\end{array}$ & Chl $a / b$ ratio \\
\hline 6 th & $14.6 \pm 1.2$ & $333.3 \pm 10.1$ & $3.7 \pm 0.1$ \\
5 th & $14.7 \pm 1.9$ & $371.7 \pm 8.3$ & $3.5 \pm 0.1$ \\
4th & $11.9 \pm 0.4$ & $268.4 \pm 11.2$ & $3.3 \pm 0.1$ \\
$3 \mathrm{rd}$ & $6.0 \pm 1.1$ & $162.8 \pm 10.6$ & $3.0 \pm 0.1$ \\
\hline
\end{tabular}

a All experiments were repeated three times independently. These values are means \pm S.D. of three independent experiments. We used about 300 leaves in one experiment.
Table 2

Levels of the thylakoid components correlated with electron transports and the PS II/ PS I ratios ${ }^{\mathrm{a}}$

\begin{tabular}{lllll}
\hline $\begin{array}{l}\text { Leaf posi- } \\
\text { tion }\end{array}$ & C-550 & P-700 & PS II/PS I & Cyt $f$ \\
& $\begin{array}{llll}\mathrm{mmol} / \mathrm{mol} \\
\text { Chl }\end{array}$ & & & $\begin{array}{l}\mathrm{mmol} / \mathrm{mol} \\
\mathrm{Chl}\end{array}$ \\
\hline 6th & $3.15 \pm 0.2$ & $2.06 \pm 0.2$ & 1.54 & $2.07 \pm 0.1$ \\
5 th & $2.80 \pm 0.2$ & $1.97 \pm 0.1$ & 1.42 & $1.66 \pm 0.2$ \\
4th & $2.30 \pm 0.2$ & $2.09 \pm 0.1$ & 1.10 & $1.52 \pm 0.1$ \\
3rd & $2.07 \pm 0.3$ & $2.11 \pm 0.2$ & 0.90 & $1.35 \pm 0.1$ \\
\hline
\end{tabular}

a All experiments were repeated three times independently. These values are means \pm S.D. of three independent experiments. We used about 300 leaves in one experiment.

the C-550 and P-700 using thylakoid membranes isolated from the 6 th to the $3 \mathrm{rd}$ leaves. McCauley and Melis [17] demonstrated that C-550 measured as the index of the PS II reaction center is the electrochromic band shift of pheophytin at $550 \mathrm{~nm}$ and this is in proportion to the amount of $Q_{\mathrm{A}}$ photoreduction. Recently, many studies have been published that C-550 is measured as amounts of the PS II reaction center $[2,25,26]$. The values obtained in this experiment are very high confidences because the value in the 6th leaves obtained here agreed with the literatures. The ratio of mmol C-550 to mol $\mathrm{Chl}$ decreased gradually from 3.2 in the 6 th leaves to 2.1 in the 3rd leaves (Table 2). In contrast, irrespective of leaf position, the levels of P-700 determined on the basis of $\mathrm{Chl}$ were approximately 2 mmol P-700 per mol Chl (Table 2). Consequently, the PS II/PS I ratio decreased from 1.5 in the 6th leaves to 0.9 in the 3 rd leaves (Table 2).

Hikosaka [2] showed that in a vine grown horizontally to avoid mutual shading, the Chl $a / b$ and PS II/PS I ratios remained constant when leaves were grown without shading. This result strongly supports the hypothesis that both ratios are affected by light intensity. Light that reaches a leaf in natural shade is weak and enriched in the far-red component of light, relative to the red component of leaves that are exposed to sunlight [27].

The light environment of the juvenile rice canopy is presented in Fig. 1. The light environment of lower leaves, which is enriched in far-red light, indicates the absorption of red light by the upper leaves. The spectral photon distributions, 
indicating an apparent increase in far-red light in the lower canopy, are shown in Fig. 1A. This region (over $750 \mathrm{~nm}$ ) is not available for photosynthesis. However, we see from Fig. 1A that the $\mathrm{red} /$ far-red $(\mathrm{R} / \mathrm{FR})$ ratio $(650 / 750 \mathrm{~nm})$ dropped from $100 \%$ at the 6 th to $19 \%$ at the 3 rd leaves. Similarly, the light intensity also decreased from $100 \%$ at the 6 th leaves to $30 \%$ at the 3 rd leaves (Fig. 1B). Thus, because these two factors change simultaneously, it is difficult to decide which is the dominant factor on the changes in the photosynthetic apparatus.

Cyt $f$ levels per unit of $\mathrm{Chl}$ decreased from 2.1 in the 6th leaves to 1.4 in the 3rd leaves (Table 2). Cyt $b_{6}$ also declined, but the extent of the decline was a little slower than that of Cyt $f$ (data not shown). This indicates that the two cytochromes degraded in the lower leaves. The observed declines in the levels of Cyt $f$ also indicate that the thylakoid components are influenced by irradiance, as plants grown in weak light had reduced levels of Cyt $f$ than did plants grown in stronger light $[5,6,23,24,28]$.

Whole chain electron transport activity, determined on the basis of Chl content, slightly decreased from the upper to the lower leaves (Table 3 ). On the other hand, the rates of electron transport activity expressed on the basis of Cyt $f$ remained fairly constant at all leaf positions (Table 3 ), implying that the Cyt $b_{6} / f$ complexes are one of the limiting factors involved in the whole chain electron transport in the lower leaves [29]. More-

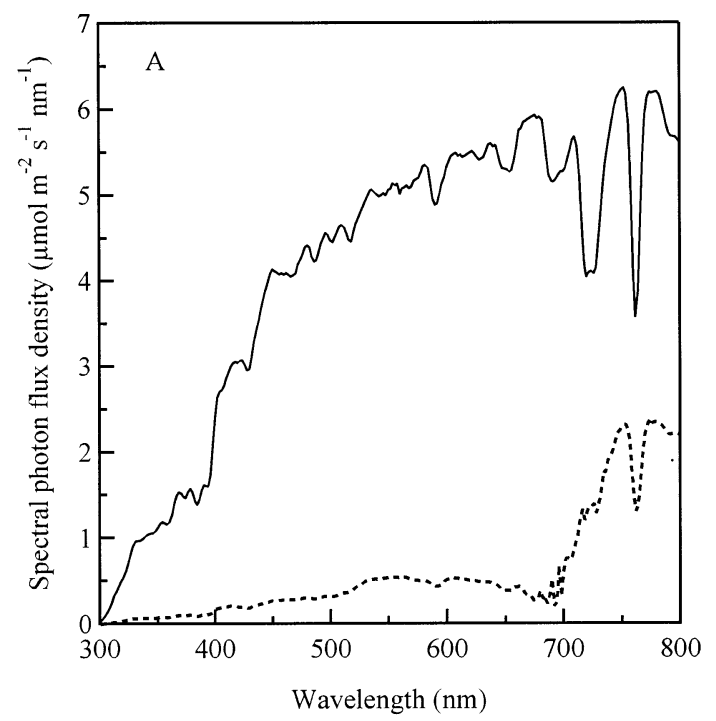

over, as shown in Table 3, there were also no significant decreases in PS II electron transport expressed on the basis of C-550 in the depth of the canopy. Chow and Anderson [30] suggested that Cyt $b_{6} / f$ complex and plastoquinone limit $P_{\max }$. Consequently, because the degradation of the thylakoid components is complex, it is not easy to decide on the site of the limitation.

As stated in the Introduction, the PS II reaction center is heterogeneous. In terms of both function and antenna size, there are two types of PS II centers, namely PS II $\alpha$, which has a large antenna and is active in electron transport, and PS II $\beta$, which has a small antenna and is unable to reduce $Q_{\mathrm{B}}[12,13]$. As a result of the large difference in antenna size, photoreduction of $Q_{\mathrm{A}}$ in $\mathrm{PS} \mathrm{II} \alpha$ proceeds more rapidly than that in PS II $\beta$ under irradiation with limited-intensity light in the DCMU-poisoned thylakoids. Thus, the percentage of abundance of PS II reaction centers can be kinetically distinguished as functional or non-functional in electron transport by measuring the rate constant of photoreduction of $Q_{\mathrm{A}}$ through growth of the area over the fluorescence induction curve.

$\mathrm{K} \alpha$ and $\mathrm{K} \beta$ stand for the rate constants of the PS II $\alpha$ and PS II $\beta$ centers, respectively, and the slope of the fast phase represents the rate constant of the $\alpha$ center, namely $\mathrm{K} \alpha$, and the slow phase represents that of the $\beta$ center, $\mathrm{K} \beta$ (Fig. 2; see refs. $[19,20])$. Table 4 shows that the relative abundance of PS II $\alpha$ was, on average, $60 \%$ of the total PS II centers in the newly developed 6th leaves. There-

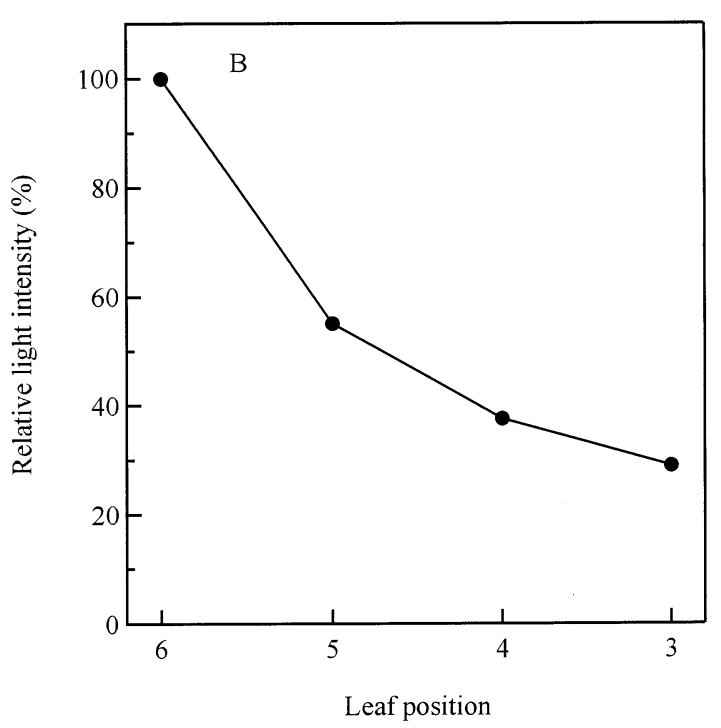

Fig. 1. The light environments of the juvenile canopy of rice seedlings. (A) Spectral photon distribution. Solid and broken lines represent at the 6th leaves and at the 3rd leaves, respectively. (B) Relative intensity of light as 100\% at the 6th leaves. 
Table 3

Whole chain electron transport activities expressed on Chl and Cyt $f$, and PS II activities expressed on C-550 ${ }^{\text {a }}$

\begin{tabular}{|c|c|c|c|}
\hline Leaf position & $\begin{array}{l}\text { PS II + PS I }\left[\mathrm{mmol} \mathrm{O}_{2}\right. \\
\left.(\mathrm{mol} \mathrm{Chl})^{(1} \mathrm{s}^{(1)}\right]\end{array}$ & $\begin{array}{l}\text { Whole chain }\left[\mathrm{mmol} \mathrm{O}_{2}\right. \\
\left.(\mathrm{mol} \text { Cyt } f)^{(1} \mathrm{s}^{(1]}\right]\end{array}$ & $\begin{array}{l}\text { PS II activity }\left[\mathrm{mmol} \mathrm{O}_{2}\right. \\
\left.(\mathrm{mol} \mathrm{C}-550)^{(1} \mathrm{S}^{(1]}\right]\end{array}$ \\
\hline 6 th & $58.4 \pm 5.5$ & $28.4 \pm 7.1$ & $29.2 \pm 8.2$ \\
\hline 4 th & $59.6 \pm 5.0$ & $39.4 \pm 6.6$ & $39.5 \pm 3.5$ \\
\hline $3 r d$ & $43.4 \pm 6.0$ & $36.1 \pm 7.9$ & $34.9 \pm 8.7$ \\
\hline
\end{tabular}

a All experiments were repeated three times independently. These values are means \pm S.D. of three independent experiments. We used about 300 leaves in one experiment.

fore, when only the PS II centers that are functional in electron transport are taken into account, the PS II $\alpha /$ PS I ratio is 0.9 in the 6 th leaves. The percentage of PS II $\alpha$ gradually increased from the top to the bottom leaves and was $70 \%$ in the 3 rd leaves. Thus, the decline in the PS II $\alpha /$ PS I ratio (Table 4) was smaller than that in the total PS II/PS I ratio (Table 2). There was also a small but significant decline in $K \alpha$ from the upper to the lower leaves. As shown in Fig. 3, $\mathrm{K} \alpha$ can be used as a measure of antenna size because $K \alpha$ increased linearly with the intensity of excitation light. In contrast, $K \beta$ did not increase linearly with light intensity but became saturated at about $100 \mu \mathrm{mol} \mathrm{m} \mathrm{m}^{-2} \mathrm{~s}^{-1}$. This implies that the rate constant of $Q_{\mathrm{A}}$ photoreduction can be used as a measure of the antenna size only in PS II $\alpha$. The antenna size of PS II $\beta$ may be estimated by measuring the initial slope of the light-response curve at a light intensity of zero, and hence the antenna size of PS II $\beta$ determined at a definite intensity of light is an underestimate. Various hypothesises at present are submitted about inactive PS II centers. Hsu and Lee [31] observed that the rate constants of slow phases of $Q_{\mathrm{A}}$ photoreduction were independent of light intensity. In their experiment, however, actinic light might have already saturated for the measurement of the PS II $\beta$. On the other hand, by using Chl $b$-less rice mutant, chlorina-2, carrying only PS II $\beta$, Terao et al. [32] showed that a half of the total PS II centers are active in electron transport by flash induced oxygen evolution. Recently, Tsurumi and Katoh revealed that the rest of the PS II centers, i.e. inactive PS II, serve as an antenna of the active PS II centers (personal communication). Therefore, because PS II $\beta$ is defined differently in various plant systems, we followed to the literatures of [32] and [33] in the present study.
The relative antenna size of PS I was determined by measuring the kinetics of P-700 photooxidation with the same excitation light as that used for fluorescence measurement (Fig. 2C, D). The rate constant of P-700 photooxidation (KI) was linear to the intensity of the excitation light (data not shown). KI decreased from the 6th to the 3 rd leaves and it was smaller than $K \alpha$, i.e. the antenna size of PS I is smaller than that of PS II $\alpha$, at all leaf positions (Table 4).

Comparing $\mathrm{K} \alpha$ with $\mathrm{KI}$, both levels decreased in parallel with leaf order from upper to lower leaves (Table 4). K $\alpha$ and the levels of C-550 per unit of Chl decreased, while the abundance of only PS II $\alpha$ increased. These results suggest that the leaves increased the total abundance of active PS II centers to compensate for a decrease in the antenna size for acclimation under light-limiting conditions. Alternatively, KI also decreased, in spite of there being no losses of P-700 per mol Chl, suggesting that leaves cause antenna size to be smaller in order to avoid receiving excess light under weak light conditions. Burkey and Wells [10] indicated that the $\mathrm{Chl}$ distribution between LHC I and LHC II was influenced by plant density. No loss of P-700 but a decrease in CP1a were observed in the dense canopy. The large shade effects in the dense canopy involved both a reduction in LHC I and an increase in LHC II, as a consequence of the shift in the light-harvesting capacity from PS I to PS II in the weak light environment. In Burkey and Wells' experiment, a dense soybean canopy indicated a high $\mathrm{FR} / \mathrm{R}$ ratio and low light intensity in the lower position. In spite of a high $\mathrm{FR} / \mathrm{R}$ ratio, its influence was low, or it had no effect. Consequently, from both their result and Fig. 1, because changes in the both factors occur synchronously in this rice canopy, it would be difficult to decide which is a main deter- 
minant of the arrangements of the photosynthetic apparatus.

The present work indicates that the $\mathrm{Chl} a / b$ and PS II/PS I ratios decreased gradually with the depth of the canopy. The most important part of this paper is that this experiment indicates that the decline in the PS II/PS I ratio resulting from the low-light environment is partly compensated for by an increase in the abundance of PS II $\alpha$ centers which are associated with a large amount of LHC II and which avoid minimally an imbalance of light absorption by the two photosystems. Thus, the decrease of the $\mathrm{Chl} a / b$ ratio paralleled the increase of PS II $\alpha$ centers. However, to drive electron transport effectively, both photosystems have to absorb light equally. Indeed, when we calculated the electron transport capacity between the two photosystems based on the equations described in [34], the capacity in the 6th leaves was 1.5-times larger than that in the 3rd leaves [35]. As a result, large imbalances occur between the two
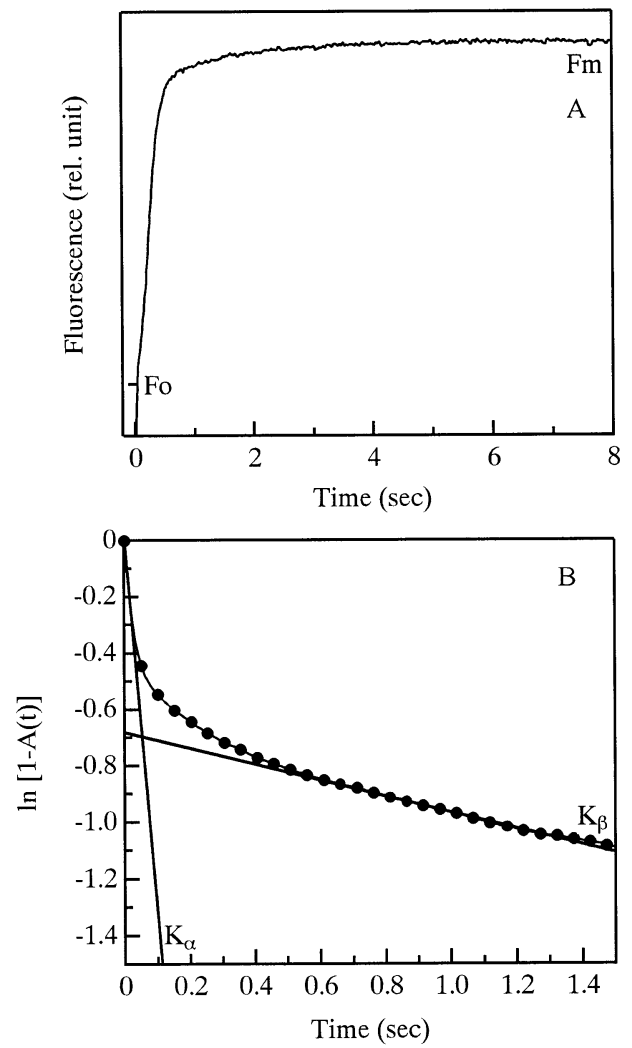

photosystems. Nevertheless we considered a state transition at first, it did not involve in the energy deployment (data not shown). Accordingly, this apparent anomaly is currently being investigated.

\section{Acknowledgements}

We express our thanks to the former Prof. Sakae Katoh for his critical comments and discussions throughout this work. We are grateful to Drs H. Ikeda and N. Matsushita, Arboricultural Research Institute, Faculty of Agriculture, Tokyo University Forests, for renting a Li-Cor model LI-1800 spectroradiometer. We also thank to $\mathrm{Mr}$ Takenobu Yamasaki for his fruitful advice and comments, and to my laboratory associates for excellent technical assistance and kind help. Finally, we would like to dedicate this paper to the mentor Prof. Sakae Katoh to commemorate his retirement from Toho University.
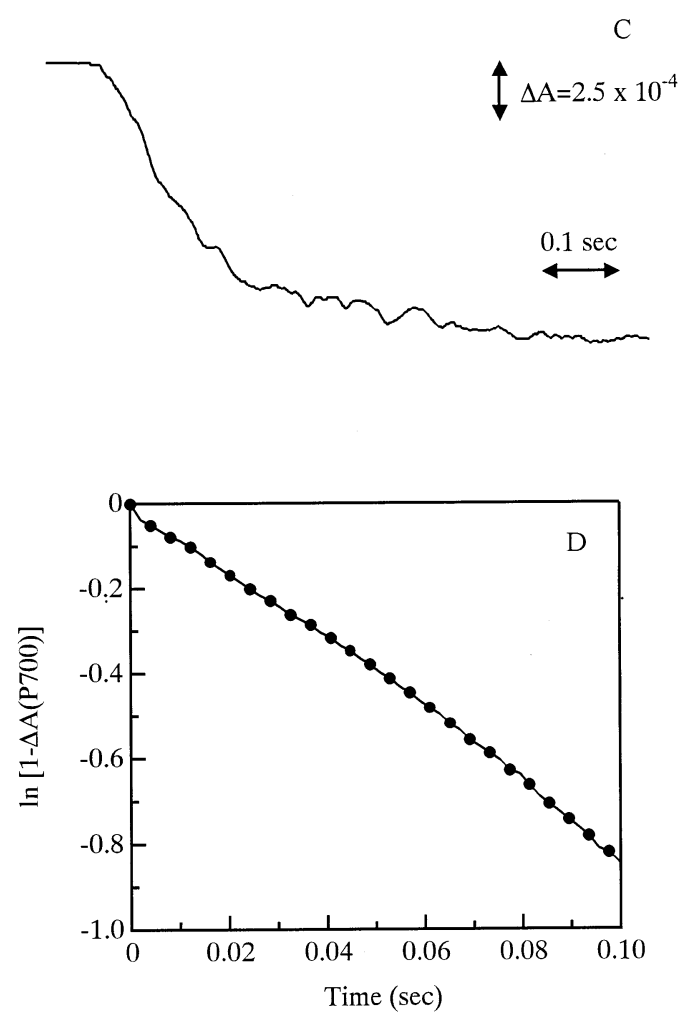

Fig. 2. (A) The fluorescence induction curve of isolated thylakoid membranes in the presence of DCMU. (B) A semilogarithmic plot of the area over the fluorescence induction curve. The slope, which defined the rate of light utilization, of slow phase represented for the rate constant of PS II $\beta$ center $(\mathrm{K} \beta)$ and of fast phase did $\mathrm{K} \alpha$. (C) The time course of P-700 photooxidation with the KCN-poisoned thylakoids. (D) A semilogarithmic plot of the absorbance change of P-700 photooxidation. 
Table 4

Relative abundances of PS II $\alpha$ and PS II $\beta$ and the rate constants of PS II $\alpha$, PS II $\beta$ and PS I in the 3 rd- 6 th leaves of the juvenile rice seedlings ${ }^{\mathrm{a}}$

\begin{tabular}{llllll}
\hline Leaf position & PS II $\alpha(\%)$ & PS II $\beta(\%)$ & K $\alpha\left(s^{-1}\right)$ & KI $\left(s^{-1}\right)$ & PS II $\alpha /$ PS I \\
\hline 6th & $60.1 \pm 0.8$ & $39.9 \pm 0.8$ & $15.7 \pm 0.6$ & $9.8 \pm 0.2$ & 0.92 \\
5th & $62.9 \pm 0.4$ & $37.1 \pm 0.4$ & $13.3 \pm 0.8$ & $8.8 \pm 0.4$ & 0.89 \\
4th & $66.2 \pm 0.5$ & $33.9 \pm 0.5$ & $12.8 \pm 0.3$ & $7.3 \pm 0.3$ & 0.73 \\
3rd & $69.9 \pm 0.6$ & $30.1 \pm 0.6$ & $11.1 \pm 0.7$ & $6.9 \pm 0.2$ & 0.69 \\
\hline
\end{tabular}

${ }^{\text {a }}$ All experiments were repeated three times independently. These values are means \pm S.D. of three independent experiments. We used about 300 leaves in one experiment.

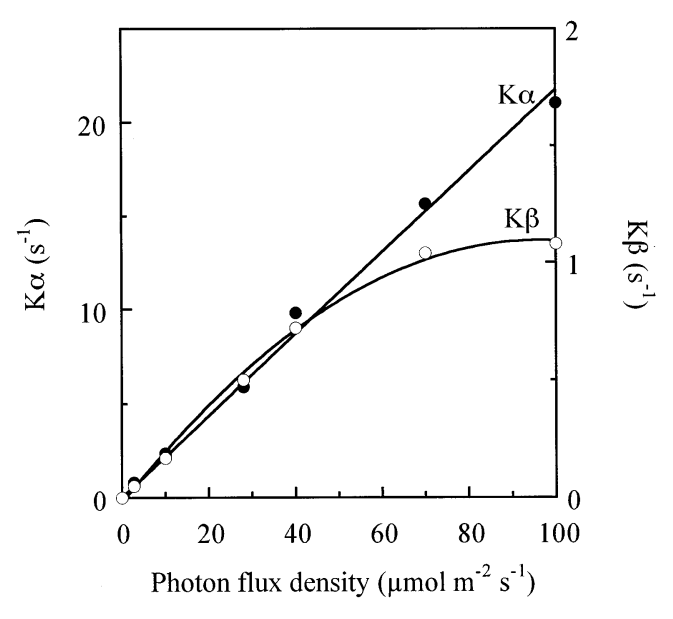

Fig. 3. Light-response curves of the rate constants of $Q_{\mathrm{A}}$ photoreduction. $\bigcirc$, the rate constants of PS II $\alpha$; $\bigcirc$, those of PS II $\beta$.

\section{References}

[1] M. Monsi, T. Saeki, Über den Lichtfaktor in den Pflanzengesellschaften und seine Bedeutung für die Stoffproduction, Jpn. J. Bot. 14 (1953) 22-52.

[2] K. Hikosaka, Effects of leaf age, nitrogen nutrition and photon flux density on the organization of the photosynthetic apparatus in leaves of a vine (Ipomoea tricolor Cav.) grown horizontally to avoid mutual shading of leaves, Planta 198 (1996) 144-150.

[3] K. Okada, S. Katoh, Two long-term effects of light that control the stability of proteins related to photosynthesis during senescence of rice leaves, Plant Cell Physiol. 39 (1998) 394-404.

[4] M. Kura-Hotta, K. Satoh, S. Katoh, Relationship between photosynthesis and chlorophyll content during leaf senescence of rice seedlings, Plant Cell Physiol. 28 (1987) $1321-1329$.

[5] T.-Y. Leong, J.M. Anderson, Adaptation of the thylakoid membranes of pea chloroplasts to light intensities. 1. Study on the distribution of chlorophyll-protein complexes, Photosynth. Res. 5 (1984) 105-115.

[6] W.R. De la Torre, K.O. Burkey, Acclimation of barley to changes in light intensity: chlorophyll organization, Photosynth. Res. 24 (1990) 117-125.

[7] A. Melis, G.W. Harvey, Regulation of photosystem stoichiometry, chlorophyll $a$ and chlorophyll $b$ content and relation to chloroplast ultrastructure, Biochim. Biophys. Acta 637 (1981) 138-145.

[8] W.-J. Lee, J. Whitmarsh, Photosynthetic apparatus of pea thylakoid membranes. Response to growth light intensity, Plant Physiol. 89 (1989) 932-940.

[9] J.H. Kim, R.E. Glick, A. Melis, Dynamics of photosystem stoichiometry adjustment by light quality in chloroplasts, Plant Physiol. 102 (1993) 181-190.

[10] K.O. Burkey, R. Wells, Effects of natural shade on soybean thylakoid membrane composition, Photosynth. Res. 50 (1996) 149-158.

[11] A. Melis, A. Murakami, J.A. Nemson, K. Aizawa, K. Ohki, Y. Fujita, Chromatic regulation in Chlamydomonas reinhardtii alters photosystem stoichiometry and improves the quantum efficiency of photosynthesis, Photosynth. Res. 47 (1996) 253-265.

[12] A. Melis, Dynamics of photosynthetic membrane composition and function, Biochim. Biophys. Acta 1058 (1991) 87-106.

[13] J. Lavergne, Two types of primary acceptors in chloroplasts photosystem II. 1. Different recombination properties, Photobiochem. Photobiophys. 3 (1982) 257-271.

[14] R.J. Porra, W.A. Thompson, P.E. Lriedemann, Determination of accurate extinction coefficients and simultaneous equations for assaying chlorophyll $a$ and $b$ extracted with four different solvents: verification of the concentration of chlorophyll standards by atomic absorption spectroscopy, Biochim. Biophys. Acta 975 (1989) 384-394.

[15] T. Hiyama, B. Ke, Different spectra and excitation coefficients of P-700, Biochim. Biophys. Acta 267 (1972) $160-171$.

[16] D.B. Knaff, D.I. Arnon, Spectral evidence for a new photoreactive component of the oxygen-evolving system in photosynthesis, Proc. Natl. Acad. Sci. USA 63 (1969) 963-969.

[17] S.W. McCauley, A. Melis, Quantitation of photosystem II in spinach chloroplasts, Biochim. Biophys. Acta 849 (1986) 175-182.

[18] D.S. Bendall, H.N. Davenport, R. Hill, Cytochrome components in chloroplasts of the higher plants, Method Enzymol. 23 (1971) 327-344.

[19] A. Melis, P.H. Homann, Kinetic analysis of the fluorescence induction in 3-(3,4-dichlorophenyl)-1,1dimethylurea poisoned chloroplasts, Photochem. Photobiol. 21 (1975) 431-437. 
[20] A. Melis, P.H. Homann, Heterogeneity of the photochemical centers in system II of chloroplasts, Photochem. Photobiol. 23 (1976) 343-350.

[21] A. Melis, Kinetics analysis of P-700 photoconversion: effect of secondary electron donation and plastocyanin inhibition, Arch. Biochem. Biophys. 217 (1982) 536-545.

[22] P. Svensson, E. Andreasson, P.-A. Albertsson, Heterogeneity among photosystem I, Biochim. Biophys. Acta 1060 (1991) 45-50.

[23] T.-Y. Leong, J.M. Anderson, Adaptation of the thylakoid membranes of pea chloroplasts to light intensities. 2. Regulation of electron transport capacities, electron carriers, coupling factor (CF1) activity and rates of photosynthesis, Photosynth. Res. 5 (1984) 117-128.

[24] W.R. De la Torre, K.O. Burkey, Acclimation of barley to changes in light intensity: photosynthetic electron transport activity and components, Photosynth. Res. 24 (1990) 127-136.

[25] S. Funayama, K. Sonoike, I. Terashima, Photosynthetic properties of leaves of Eupatorium makinoi infected by a geminivirus, Photosynth. Res. 53 (1997) 253-261.

[26] K. Hikosaka, I. Terashima, Nitrogen partitioning among photosynthetic components and its consequence in sun and shade plants, Func. Ecol. 10 (1996) 335-343.

[27] M.C. Rousseaux, A.J. Hall, R.A. Sánchez, Far-red enrichment and photosynthetically active radiation level influence leaf senescence in field-grown sunflower, Physiol. Plant. 96 (1996) 217-224.

[28] E.H. Murchie, P. Horton, Contrasting patterns of photosynthetic acclimation to the light environment are depen- dent on the differential expression of the responses to altered irradiance and spectral quality, Plant, Cell Environ. 21 (1998) 139-148.

[29] P.J. Holloway, D.J. Maclean, Rate-limiting steps of electron transport in chloroplasts during ontogeny and senescence of barley, Plant Physiol. 72 (1983) 795-801.

[30] W.S. Chow, J.M. Anderson, Photosynthetic responses of Pisum sativum to an increase in irradiance during growth 1. Photosynthetic activities, Aust. J. Plant Physiol. 14 (1987) $1-8$.

[31] B.-D. Hsu, J.-Y. Lee, A study on the fluorescence induction curve of the DCMU-poisoned chloroplast, Biochim. Biophys. Acta 1056 (1991) 285-292.

[32] T. Terao, K. Sonoike, J. Yamazaki, Y. Kamimura, S. Katoh, Stoichiometries of photosystem I and photosystem II in rice mutants differently deficient in chlorophyll b, Plant Cell Physiol. 37 (1996) 299-306.

[33] A. Melis, Functional properties of photosystem II $\beta$ in spinach chloroplasts, Biochim. Biophys. Acta 808 (1985) 334-342.

[34] A. Melis, J.M. Anderson, Structural and functional organization of the photosystems in spinach chloroplasts. Antenna size, relative electron-transport capacity, and chlorophyll composition, Biochim. Biophys. Acta 724 (1983) 473-484.

[35] J. Yamazaki, Y. Kamimura, Y. Sugimura, Changes in photosynthetic apparatus in the juvenile rice canopy and a possible function of photosystem $I$ in the bottom leaves, Z. Naturforsch. 54c (1999) in press. 